\title{
Resecabilidad y mortalidad operatoria de la gastrectomía subtotal y total en pacientes con cáncer gástrico avanzado, entre 1969 y 2004
}

\author{
Attila Csendes J, Patricio Burdiles P, Italo Braghetto $M$, \\ Juan Carlos Díaz J, Fernando Maluenda G, 0 wen Korn B, \\ G uillermo Watkins $\mathbf{S}$, Jorge Rojas $\mathrm{C}$. \\ Evolution of resectability and \\ mortality rates of total and subtotal \\ gastrectomy for gastric cancer
}

\begin{abstract}
Background: The only curative treatment for gastric cancer is its surgical excision associated to a lymph node dissection. Aim: To study the evolution of resectability and operative mortality of total and subtotal gastrectomy for gastric cancer, in a period of 35 years. Material and methods: Review of medical records of 3000 patients with gastric cancer, operated between 1969 and 2004. Resectability and mortality of total and subtotal gastrectomy was compared in four successive periods (1969 to 1979, 1980 to 1989, 1990 to 1999 and 2000 to 2004). Results: In the four periods there was a steady and significant increase in resectability rate from 49 to $85 \%$. Mortality of total and subtotal gastrectomy decreased significantly from 17 to $2 \%$ and from 25 to $1 \%$, respectively. Conclusions: Resectability and mortality rates of total and subtotal gastrectomy have improved with time. Probably a better pre and postoperative care and the experience of the surgical team have an influence in this favorable change (Rev Méd Chile 2006; 134: 426-32).

(Key words: Gastrectomy; Stomach neoplasms; Surgical procedures, operative)
\end{abstract}

Recibido el 10 de junio, 2005. Aceptado el 1 de septiembre, 2005.

Departamento de Cirugía, Hospital Clínico de la Universidad de Chile. Santiago de Chile.

El cáncer gástrico sigue siendo la causa más importante de mortalidad por tumores malignos en Chile. Su tasa anual se ha mantenido en los últimos años cercana a $20 \times 100.000$ habitantes, $\mathrm{y}$

Correspondencia a: Attila Csendes, MD, FACS (Hon). Director, Departamento de Cirugía, Hospital Clínico JJ Aguirre. Santos Dumont 999. Santiago, Chile. Fono: 56-2-7774387. Fax: 56-2-7775043. E mail: acsendes@med.uchile.cl es responsable de cerca de 3.000 fallecidos por año ${ }^{1}$. Por lo tanto, representa un serio problema de salud pública. El único tratamiento que puede lograr curación y sobrevida prolongada es, hasta ahora, la resección oncológica² ${ }^{2}$. Sin embargo, este procedimiento tiene riesgos (complicaciones y mortalidad operatoria) y la resecabilidad del tumor es variable de un centro a otro y de acuerdo al estado de la enfermedad. 
El presente estudio analiza las cifras de resecabilidad y de mortalidad operatoria para gastrectomía subtotal y total, en un periodo de 35 años, en un centro universitario de referencia, con gran número de cirugías de alta complejidad.

\section{Material y MÉTOdo}

En este estudio retrospectivo y descriptivo se incluyeron a todos los pacientes operados entre 1969 y 2004 en el Departamento de Cirugía del Hospital Clínico de la Universidad de Chile. Los pacientes del lapso entre 1969 y 1979 corresponden a un estudio cooperativo interhospitalario, en que el Hospital Clínico Universitario fue el primer participante ${ }^{3}$. Todos los pacientes tenían un diagnóstico completo preoperatorio con endoscopia y biopsias, confirmando la presencia de un adenocarcinoma gástrico, salvo en el lapso 1969-1979, en que la endoscopia se realizó sólo en $65 \%$ de los pacientes. Se excluyeron pacientes sometidos a gastrectomía por linfoma gástrico, por lesiones benignas, o con cáncer del muñón gástrico. Pacientes con cáncer gástrico incipiente también fueron excluidos. Para el presente estudio, sólo se consideraron dos puntos muy precisos y finales. La resecabilidad del tumor, es decir, el porcentaje de pacientes operados en los que se pudo resecar el tumor, y la mortalidad operatoria, es decir, los fallecidos hasta 30 días después de la cirugía. Sin embargo, si el paciente permanecía hospitalizado después de 30 días y fallecía con posterioridad sin irse de alta, también se consideró como mortalidad operatoria.
Hasta 1990, la técnica operatoria consistió básicamente en una gastrectomía subtotal D2 con omentectomía y reconstrucción tipo Billroth II. Desde esa fecha se emplea una anastomosis con asa en Y-de- Roux de $70 \mathrm{~cm}$ de largo. La gastrectomía total fue D2 con omentectomía y reconstrucción con asa en Y-de-Roux en todos ${ }^{2-8}$.

Para los cálculos estadísticos, se empleó el test exacto de Fisher, tomando un valor $\mathrm{p}<0,05$ como significativo.

\section{RESULTADOS}

En la Tabla 1 se muestra la resecabilidad del cáncer gástrico en 4 periodos sucesivos. Se aprecia un aumento progresivo y significativo de la tasa de resecabilidad en estos pacientes ( $p<0,001$ ).

La Tabla 2 señala la mortalidad operatoria de pacientes con cáncer gástrico sometidos a gastrectomía subtotal. Se aprecia una disminución de la mortalidad, desde $17 \%$ a una cifra cercana a $2 \%$ ( $p<0,001)$. La Tabla 3 describe la mortalidad operatoria de los pacientes sometidos a gastrectomía total. Se aprecia una disminución desde casi $25 \%$ a $0,8 \%$ ( $p<0,0001$ ). En ambas tablas se muestra el porcentaje relativo de cada operación durante los periodos del estudio. Se aprecia que en el primer periodo, la gastrectomía subtotal correspondió a $64 \%$ del total de las resecciones gástricas, mientras que en el último periodo, sólo correspondió a $25 \%$, lo que es una disminución muy significativa $(p<0,001)$. Por otra parte, la gastrectomía total aumentó de $36 \%$ en el primer periodo a $75 \%$ en el último periodo ( $p<0,001)$.

Tabla 1. Resecabilidad del cáncer gástrico (1969-2004)

\begin{tabular}{|lcrr|}
\hline Periodo (años) & Total operados & \multicolumn{2}{c|}{ Resecabilidad } \\
& & $\mathrm{n}$ & $(\%)$ \\
\hline $1969-1979$ & 1.242 & 606 & $(48,7)$ \\
$1980-1989$ & 612 & 444 & $(72,5)$ \\
$1990-1999$ & 748 & 591 & $(79)$ \\
$2000-2004$ & 398 & 337 & $(85)$ \\
Total & 3.000 & 1.978 & $(65,9)$ \\
\hline
\end{tabular}


Tabla 3. M ortalidad operatoria de la gastrectomía total (1969-2004)

\begin{tabular}{|c|c|c|c|}
\hline Periodo (años) & $\mathrm{n}$ & $\begin{array}{l}\text { Mortalidad } \\
\mathrm{n} \quad(\%)\end{array}$ & $\%$ Total resecados \\
\hline 1969-1979 & 219 & $54 \quad(24,6)$ & 36,1 \\
\hline 1980-1989 & 220 & $31 \quad(14)$ & 49,6 \\
\hline 1990-1999 & 340 & $11 \quad(3,2)$ & 57,5 \\
\hline $2000-2004$ & 252 & $(0,8)$ & 74,8 \\
\hline
\end{tabular}

Tabla 2. M ortalidad operatoria de la gastrectomía subtotal (1969-2004)

\begin{tabular}{|lcccc|}
\hline Periodo (años) & $\mathrm{n}$ & \multicolumn{2}{c|}{$\begin{array}{c}\text { Mortalidad } \\
\text { (\%) }\end{array}$} & \% Total resecados \\
\hline $1969-1979$ & 387 & 66 & $(17)$ & 63,9 \\
$1980-1989$ & 224 & 7 & $(3,1)$ & 50,4 \\
$1990-1999$ & 251 & 5 & $(2,0)$ & 42,5 \\
$2000-2004$ & 85 & 2 & $(2,3)$ & 25,2 \\
\hline
\end{tabular}

\section{Discusión}

El presente estudio muestra un notable avance en el manejo de pacientes con cáncer gástrico. Hemos incluido sólo el análisis de 2 puntos finales que son decisivos en el pronóstico de un paciente: la resecabilidad, es decir, la probabilidad de poder someterse a una resección quirúrgica, que es la única alternativa de sobrevida que tiene el paciente, y el riesgo que tiene esta resecabilidad, es decir, la probabilidad de fallecer después de la operación a causa de alguna complicación médica o quirúrgica. No fue nuestro propósito analizar la sobrevida a largo plazo, que ha sido publicada previamente con gran detalle ${ }^{7,8}$. El significativo aumento de la resecabilidad del cáncer gástrico ya había sido publicado como uno de los progresos importantes que se había logrado en el manejo de pacientes con cáncer gástrico ${ }^{9}$. Hay múltiples explicaciones para este aumento: por una parte, la expansión de métodos de diagnóstico masivo como la endoscopia, que ha permitido su acceso a toda la población. Por otra parte, la consulta más precoz, producto de una continua educación tanto de la población como de los médicos generales. Ya casi no vemos pacientes desnutridos, caquécticos, que lleguen de esta manera a la primera consulta y el aspecto nutritivo de estos pacientes no está tan comprometido como en décadas previas. Otro aspecto que hay que destacar en la última década, es la masificación de un completo estudio preoperatorio, que incluye un escáner abdominal de rutina. Por lo tanto, se puede excluir a los pacientes con evidentes metástasis hepáticas, peritoneales o en otros órganos, ya que en décadas pasadas a casi todos se sometía a una laparotomía exploradora, por falta de otros métodos de diagnóstico preoperatorios ${ }^{10}$. Los métodos de imágenes actuales permiten seleccionar mucho mejor a los pacientes candidatos a cirugía. Sin embargo, aun en años recientes (2000 a 2004) se aprecia que $15 \%$ de los pacientes termina en una laparotomía o laparoscopia exploradora ${ }^{11}$.

Respecto a la técnica quinúrgica y a la mortalidad operatoria, se aprecia en las Tablas 2 y 3 un cambio importante en la proporción relativa de la gastrectomía total versus subtotal: mientras entre 1969 y 1979 la gastrectomía subtotal comespondió al $64 \%$ de la 
resección gástrica, entre 2000 y 2004, la gastrectomía total correspondió a $75 \%$ de las resecciones gástricas. Este significativo aumento de la gastrectomía total de $36 \%$ a $75 \%$ ya había sido advertido previamente ${ }^{9}$. Este cambio se explica básicamente por el hecho que el cáncer gástrico en la década del 60 a 70 se ubicaba en casi $70 \%$ en el tercio inferior o distal, mientras que en la actualidad en $70 \%$ se ubica en el tercio superior o proximal del estómago. La notable mejonía de la mortalidad de la gastrectomía total, que en la actualidad es cercana al 1\%, tiene varias explicaciones «secundarias»y sprimarias». Entre las que llamamos «secundarias», pero que en realidad tienen una enorme importancia, se puede mencionar una mejoría de la evaluación y el tratamiento nutricional de los pacientes, un mejor manejo kinésico pre y postoperatorio, más complejas y eficaces unidades de tratamiento intermedio e intensivo, una notable mejoría del manejo anestésico, y el empleo de medicamentos y antibióticos de gran eficacia. Estos aspectos son válidos para todo tipo de cirugía. Sin embargo, los motivos que llamamos primarios» para explicar las cifras actuales de morbimortalidad están en el «efecto cirujano». Esto se refiere a la experiencia del cirujano, a la dedicación a esta patología, a la posibilidad de ser atendidos por cirujanos de «alto volumen»en hospitales de «alto volumen» Esto significa que el grupo quirúrgico dedicado a la atención de pacientes con cáncer gástrico debe tener un débito anual de pacientes que les permita tener una importante experiencia. Esto se ha reflejado claramente en 2 publicaciones internacionales y en la presente serie. Mc Cullach, en 1994, evaluó a 5 diferentes cirujanos, realizando preferentemente una gastrectomía subtotal y en $10 \%$ de los pacientes una gastrectomía total ${ }^{12}$. La mortalidad operatoria fue muy diferente entre uno y otro cirujano, variando de 0 a 35\%. Fujita, en 2002, analizó las complicaciones postoperatorias de cirujanos «de alto y bajo volumen» en operaciones de cáncer gástrico, demostrando una tasa de $11 \%$ de complicaciones para el primer grupo y de $24 \%$ para el segundo grupo $(p<0,04)^{13}$. En el presente estudio se reproduce lo mismo. En la década 1970 - 79 había numerosos cirujanos que operaban cáncer gástrico, varios de ellos realizando menos de 5 operaciones por año, con una mortalidad global para la gastrectomía total de $25 \%^{10}$. Con el transcurrir del tiempo, esta cirugía quedó en manos de un reducido grupo de cirujanos, todos con amplia experiencia y con alto volumen de operaciones anuales, con una mortalidad actual menor a 1\%. La pregunta es jpor qué sucede esto? Porque, fuera de todos los progresos detallados en los factores «ecundarios», el grupo quirúrgico con experiencia puede manejar mejor a los pacientes en el pabellón y en el periodo postoperatorio. La experiencia quirúrgica significa un manejo adecuado de los tejidos, rapidez en el acto quirúrgico, un protocolo estándar de técnica quirúrgica, igual para todos los pacientes. Además, con la experiencia se pueden realizar diagnósticos más precoces y oportunos de alguna complicación postoperatoria. En la Tabla 4 se muestra la mortalidad operatoria de algunos grupos quirúrgicos chile-

\section{Tabla 4. M ortalidad operatoria de la gastrectomía total de autores chilenos en la última década (1995 a 2004)}

\begin{tabular}{|llcc|}
\hline $\begin{array}{l}\text { Autor } \\
\text { (referencias) }\end{array}$ & Año & № casos & $\begin{array}{c}\text { Mortalidad operatoria } \\
\%\end{array}$ \\
\hline Alvarez $^{14}$ & 1995 & 104 & 5,7 \\
Bustamante $^{15}$ & 1995 & 66 & 9,1 \\
Venturelli $^{16}$ & 1996 & 60 & 2,0 \\
Burmeister $^{17}$ & 1997 & 546 & 1,6 \\
Cenitagoya $^{18}$ & 1997 & 134 & 9,5 \\
Hernández $^{19}$ & 1999 & 102 & 10,7 \\
Alvarez $^{20}$ & 2004 & $163<65$ años & 2,5 \\
Valenzuela $^{21}$ & & $101>65$ años & 6,9 \\
\hline
\end{tabular}


nos con gran experiencia en este tema ${ }^{14-21}$. En la Tabla 5 se muestran los resultados de diferentes grupos quirúrgicos internacionales que han realizado gastrectomía total por cáncer gástrico, en estudios randomizados incluyendo un estudio nuestro ${ }^{22-29}$. Se observan importantes variaciones en el riesgo operatorio, dependiendo de la experiencia y dedicación de cada grupo. Un reciente análisis de la Cochrane Library, referente a resección gástrica por cáncer gástrico, concluye que esta cirugía debiera realizarse, en lo posible, por cirujanos de amplia experiencia que se hayan entrenado especialmente y que puedan mostrar, a través de una auditonía personal, cifras de mortalidad aceptables ${ }^{30}$. Una manera muy objetiva de demostrar entre nosotros el (efecto quirúrgico primario», es analizar la tasa de fístulas anastomóticas tipo I y II. En una publicación de 1990 hubo 8,8\% de fístulas tipo I y 7,8\% de tipo $\mathrm{II}^{30}$. Estas últimas corresponden a las fístulas clínicas, con cuadro séptico, que requieren un intensivo manejo médico y quirúrgico y eventual reoperación. La mortalidad de las fístulas tipo II en esa época era de 70\%. En la década previa, entre 1970 y 1980, la incidencia de fístula anastomótica tipo II era 14\%, con una elevada mortalidad 3 ,10. En esa época, señalábamos que nuestra meta era llegar a una mortalidad de 5 a $7 \%^{3}$. Ahora, con una incidencia de fístula anastomótica menor a $2 \%$ (por la dedicación y experiencia del grupo quinúrgico) y una mortalidad menor a $1 \%$, es la mejor cifra a que podemos llegar y creemos que es imposible mejorar, en las actuales condiciones.
Es importante, finalmente, detallar el actual protocolo de tratamiento, que es absolutamente estándar desde hace más de 10 años. Los pacientes ingresan el día previo a la cirugía, colocándole en pabellón, por anestesistas, una vía venosa central y un catéter epidural. Los pacientes son evaluados y conocidos por la unidad de nutrición intensiva de nuestro departamento, para su manejo pre y postoperatorio ${ }^{32}$. También son tratados por la unidad de kinesiterapia. En el pabellón, se colocan vendas compresivas y elásticas, con un agregado de compresión intermitente venosa externa en ambas extremidades inferiores. Se colocan una sonda Foley vesical y $1 \mathrm{~g}$ de cefazolina intravenosa. La cirugía estándar es una gastrectomía total, omentectomía mayor y menor, disección linfonodal D2 (que incluye las barreras 1 hasta 13), sin pancreatectomía, con esplenectomía en forma excepcional, como lo mostramos en nuestro estudio prospectivo y randomizado ${ }^{29}$. La anastomosis esófago-yeyunal se realiza con sutura mecánica con stapler circular 25 (Tyco Healthcare), con refuerzo seromuscular a puntos separados de Biosyn 3-0 (Tyco Healthcare). Se colocan 2 drenajes perianastomóticos. En el postoperatorio se emplea rutinariamente heparina en dosis de $5.000 \mathrm{ml} \mathrm{c} / 8 \mathrm{~h}$, vendas elásticas, levantada precoz. La sonda vesical se retira a las $48 \mathrm{~h}$ y el catéter epidural a las $72 \mathrm{~h}$. La kinesiterapia respiratoria se realiza 2 a 3 veces al día. La nutrición enteral, a través de una sonda nasoyeyunal colocada durante la operación, se comienza a las 24 a 48 h y se

\section{Tabla 5. M ortalidad operatoria de la gastrectomía total D 2 en autores extranjeros (Estudios prospectivos y randomizados)}

\begin{tabular}{|lccc|}
\hline $\begin{array}{l}\text { Autor } \\
\text { (referencias) }\end{array}$ & Año & o casos & $\begin{array}{c}\text { Mortalidad operatoria } \\
\%\end{array}$ \\
\hline Gouzi $^{22}$ & 1989 & 93 & 1,3 \\
Roberston $^{23}$ & 1994 & 29 & 3,4 \\
Bonenkamp $^{24}$ & 1995 & 331 & 10 \\
Cuschieri $^{25}$ & 1996 & 200 & 13 \\
Bozzetti $^{26}$ & 1999 & 302 & 2,3 \\
DeGiuli $^{27}$ & 2004 & 86 & 1 \\
Wu $^{28}$ & 2004 & 111 & 0 \\
Csendes $^{29}$ & 2002 & 187 & 3 \\
\hline
\end{tabular}


mantiene por 5 días, promedio ${ }^{32}$. Alrededor del $6^{0}$ o 70 día, se realiza un control radiológico con bario de la anastomosis. Si está normal (lo que ocurre en $98 \%$ de los casos), se retira la sonda nasoyeyunal y se comienza alimentación oral. El alta médica del hospital es alrededor del 8o día postoperatorio.

\section{REFERENCIAS}

1. Instituto Nacional de Estadísticas. Anuario de Estadísticas Vitales 2000

2. CSENDEs A. Estado actual del tratamiento de cáncer gástrico. Rol de la linfadenectomía. Cuad Cir 1994; 8: 82-91.

3. Csendes A, Caracci M, Parr G, Pavez J, Venturem A. Aspectos clínicos y terapéuticos del cáncer gástrico. 1973-1979. Rev Méd Chile 1983; 111: 262-7.

4. Amat J, Pérez P, Csendes A, Cardemil G. Gastrectomía total. Rev Chil Cir 1976; 28: 109-12.

5. Csendes A, Braghetto I, Burdiles P, Debandi A, Díaz JC, RAPAAPORT J ET AL. Estudio prospectivo y randomizado de 2 tipos de anastomosis como método de reconstrucción después de gastrectomía total. Rev Chil Cir 1994; 46: 348-55.

6. Csendes A, Korn O, Burdiles P, Braghetto I, Díaz JC, MaluENda F. Comparación de la anastomosis esofagoyeyunal mecánica versus normal para gastrectomía en pacientes con cáncer gástrico. Rev Chil Cir 1994; 46: 59-62.

7. Csendes A, Medina E, Smok G, Korn O. Sobrevida a largo plazo de pacientes con cáncer gástrico sometidos a gastrectomía subtotal. Rev Méd Chile 1993; 121: 1388-94.

8. CSENDES A. Cáncer gástrico. Estado actual de su tratamiento y resultados a largo plazo. Rev Chil Cir 1992; 44: 260-7.

9. Csendes A, Smok G, Medina S, Salgado I, Rivera R, Quitral M. Caractenísticas evolutivas del cáncer gástrico 1958-1990. Rev Méd Chile 1992; 120: 36-42.

10. Csendes A, Flores N. Cáncer gástrico. Estudio cooperativo interhospitalario II parte. Tratamiento, hallazgos anatomoquirúrgicos y evolución postoperatoria. Arch Soc Cir Chil 1975; 37: 302-8.

11. Yarmuch J, Amat J, Csendes A, Rojas J, Maluenda F, BuRdiles P ET AL. Laparoscopia diagnóstica en
Este estricto protocolo y la permanente dedicación a estos enfermos nos han permitido llegar a estas cifras de morbimortalidad. Pensamos que es posible aplicar estos mínimos estándares a lo largo de Chile y optimizar los resultados inmediatos del tratamiento quirúrgico del cáncer gástrico.

algunos cánceres digestivos. Rev Chil Cir 1999; 51: 401-4.

12. Mc СuшасH P. Should general surgeons treat gastric carcinoma? An audit of practice and results, 1980-1985. Br J Surg 1994; 81: 417-20.

13. FuJITA T, YAMAZAKI Y. Influence of surgeons volume or early outcome after total gastrectomy. Eur J Surg 2002; 168: 535-8.

14. Alvarez R, Damayo A, Funke R, Pozo R, CastiLo M, KiAASEN R ET AL. Gastrectomía total ampliada. Rev Chil Cir 1995; 47: 41-8.

15. Bustamante M. Fístula anastomótica en gastrectomía total por cáncer. Rev Chil Cir 1995; 47: 12836.

16. Venturem A, Mivea A, Díaz J, Kuschel C, Hermosila P, AvenDaÑo R. Esofagoyeyunoanastomosis con sutura continua en gastrectomía total por cáncer gástrico. Rev Chil Cir 1996; 48: 348-51.

17. Burmeister R, García C, Benavides C, Apablaza S. Fístulas de las anastomosis esofagoyeyunales. Experiencia de 10 años. Rev Chil Cir 1997; 49: 402-5.

18. Cenitagoya G, Bergh C, Kunger J, Casanova D. Cáncer gástrico. Sobrevida real a 5 años. Rev Chil Cir 1997; 49: 626-32.

19. Hernández F, Matus C, Cerda R, Alvarez S, Leiva L, CoRTÉs L ET AL. Gastrectomía total en cáncer gástrico. Resultados inmediatos. Rev Chil Cir 1999; 51: 259-64.

20. Alvarez R, Funke R, Molina H, Cancino A, Torres D, Dinan R. Gastrectomía total en pacientes mayores de 65 años. Rev Chil Cir 2004; 56: 443-8.

21. Valenzueia L, Martínez R, Sharp A, Naguira C, Roblero HL. Interposición de asa tipo HenleyLongmine en gastrectomía total. Rev Chil Cir 2004; 56: 449-51.

22. Gouzi JL, Huguier M, Fagniez PL, Launois B, Flamant Y, Lacaine F ET al. Total versus subtotal 
gastrectomy for adenocarcinoma of the gastric antrum. Ann Surg 1989; 209: 162-6.

23. Roberston CS, Chung SC, Woods SD, Griffin SM, Raimes SA, Lau JT et al. A prospective randomized trial comparing R1 subtotal gastrectomy with R3 total gastrectomy for antral cancer. Ann Surg 1994; 220: 176-82.

24. Bonenkamp JJ, Songun I, Hermans J, Sasako M, WeivaArt K, PenkKer JT et al. Randomized comparison of morbidity after D1 and D2 dissection for gastric cancer in 996 Dutch patients. Lancet 1995; 345: 745-8.

25. Cuschieri A, Fayers P, Fielding J, Craven J, BanCEWICZ J, Joy PV ET AL. Postoperative morbidity and mortality after D1 and D2 resections for gastric cancer. Preliminary results of the MRC randomized controlled surgical trial. Lancet 1996; 347: 995-9.

26. Bozzetti F, Manubani E, Bonfanti G, Miceli R, Piano C, GenNari L. Subtotal versus total gastrectomy plus gastric cancer: Five years survival rates in a multicenter randomized Italian trial. Ann Surg 1999; 230: 170-1.

27. DeGiuli M, Sasako M, Calgaro M, Garino $M$, REBECCHI F. Morbidity and mortality after D1 and D2 gastrectomy for cancer: Interior analysis of the Italian gastric cancer study group (IGCSG) randomized surgical trial. Eur J Surg Oncol 2004; 30: 303-5.

28. Wu CW, Hsiung CA, Lo SS, Hsieh MC, Shia LT, Whang-Peng J. Randomized clinical trial of morbidity after D1 and D3 surgery for gastric cancer. $\mathrm{Br}$ J Surg 2004; 91: 283-7.

29. Csendes A, Burdiles P, Rojas J, Braghetto I, Díaz JC, Maluenda F. A prospective randomized study comparing D2 total gastrectomy versus D2 total gastrectomy plus splenectomy in 187 patients with gastric carcinoma. Surgery 2002; 131: 401-7.

30. McCuLoch P, Nita ME, Kazi H, Gama-Rodríguez J. Extended versus limited lymph node dissection technique for adenocarcinoma of the stomach. The Cochrane Library, Issue 2; 2005. Oxford Update.

31. Csendes A, Díaz JC, Burdiles P, Braghetto I, Maluenda F, Nava O et al. Classification and treatment of anastomotic leakages after extended total gastrectomy in gastric carcinoma. Hepatogastroenterology 1990; 37: 174-7.

32. Papapietro K, Díaz E, Csendes A, Díaz JC, Burdiles $P$, Maluenda $F$ et al. Nutrición enteral precoz en pacientes con gastrectomía total por cáncer gástrico. Rev Méd Chile 2002; 130: 1125-30. 